ORIGINAL PROF-2069

\title{
RADIOLOGY REQUEST FORM;
}

A neglected medical document

Dr. Abdus Salam, Dr. Saif-ud-din Saif

ABSTRACT.... Background: An incompletely filled Radiology Request Form (RRF) is a common problem faced by both radiologists and radiographers. Objective: The study was carried out to objectively evaluate the adequacy of completion of radiology request forms in a tertiary care centre. Indoor and outdoor patient departments of POF Hospital Wah Cantonment. Design: Descriptive, retrospective study. Setting: Radiology Department POF Hospital, Wah Cantonment. Period: 01 Jul 2009 to 01 September 2009. Methods: A total of 1500 request forms received by the radiology department from 01Jul 2009 to 01 Sep 2009 were reviewed. These included requests for a variety of examinations from different departments within POF Hospital, Wah Cantonment. A database of the collected forms was created, noting which of the various fields were adequately completed. Results: Only 270 out of the 1500 forms were completed in full and 1230 were not completely filled. The only parameter fulfilled in all the forms was the presence of referring doctor's signature. The commonest blank fields were as follows: patient location: $62 \%$, clinical notes: $67.26 \%$, doctor's name: $47.33 \%$ and date of referral: $14.2 \%$. Conclusions: The inadequate transmission of clinical information observed in this study is typical example of the various problems that radiologists have to face.

Key words: Radiology request forms, incomplete request forms, Clinical information

Article Citation

Salam A, Saif S. Radiology request form; A neglected medical document. Professional Med J 2013;20(2): 308-312.

\section{INTRODUCTION}

Good communication between radiologists and referring physicians is a vital aspect of optimal health care. Without smooth information flow, even the latest technological innovations in medicine may be useless. This need can be illustrated in requesting radiology examination. If there is a lack of proper communication in the form of inadequate clinical history of the patient, then the radiologist may perform an unnecessary procedure or perform the inappropriate procedure. Lack of clinical history on radiology requisition is a universal problem ${ }^{1}$. Several studies identify a large number of problems in the quality of radiology requisitions ${ }^{2}$. Inadequate communication leads to suboptimal quality of care for the patient.

Most hospitals in Pakistan use paper forms to request radiology examinations. With current technological advances to expedite the ordering process, modern hospitals are beginning to use electronic forms in Hospital Information System. An ordering physician may use a computer terminal to send the request. The radiology department instantaneously receives the request form and performs the appropriate examination in a timely manner. This electronic ordering system reduces the amount of time and paper wasted and increases the efficiency of the requesting process.

\section{MATERIAL AND METHODS}

In assessing local practice, 1500 randomly selected request forms were scrutinized at the radiology department, POF Hospital, Wah Cantonment, during 01 Jul 2009 to 01 September 2009. The specimen requisition form used in the subject hospital is given as table-I. This hospital is a tertiary care centre imparting teaching to under graduate and post graduate students. The request forms were selected by convenience sampling method to avoid the bias. They included a balanced variety of requests for an array of examinations including Plain Radiography and Fluoroscopic Radiography. They included referrals from different departments both from a ward setting, as well as from the Outpatients clinics. Referrals from private practitioners were not included. For each form, the presence or absence of adequate information in the 
appropriate field was observed. The database subsequently obtained was analyzed.

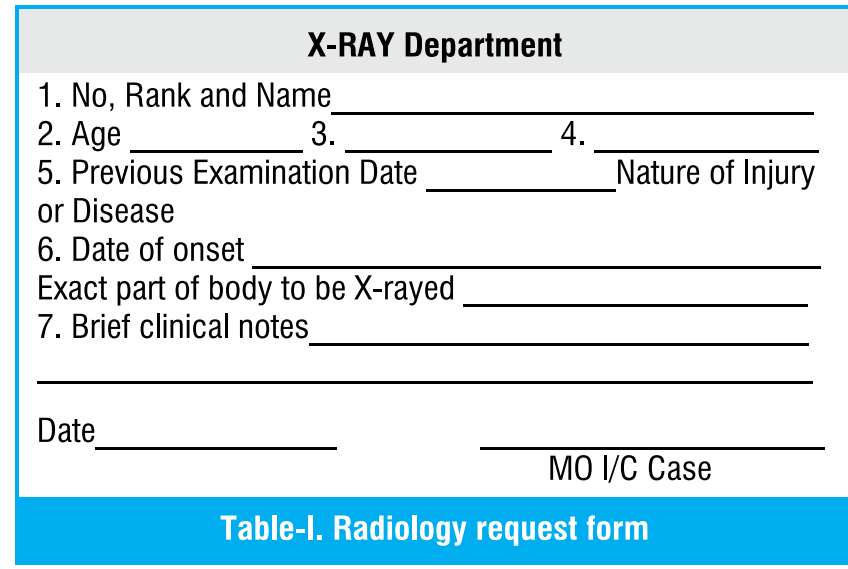

\section{RESULTS}

The standard clearly states that ALL radiology request forms should be adequately completed. Our audit's data analysis revealed that only 270 out of the 1500 forms reviewed were completed in full. The only parameter fulfilled in all the forms was the presence of signature of the referring doctor's signature. The location (ward/OPD) was mentioned in 931 forms, and the signing doctor's name was evident in 710. Only one form had doctor's phone number in the stamp. MO I/C stamp without doctor's name was used in 213 cases and 59 forms were without doctor's stamp. The patient's unit/address was provided in 1476 forms, the date in 213 and the patient's age in 1488 forms. The clinical background field was filled in 1009 forms. This figure is much better than an earlier secondary care based hospital study where clinical information was provided in $50 \%$ of the cases ${ }^{3}$.

In 610 forms patient's name was not mentioned rather son of / daughter of / wife of / father of written with reference to the employees. A chart depicting the percentages of completion of the various fields is given in Table III. In 39 cases, laboratory request forms were used for making radiology request. The medical officers (690) followed by postgraduate trainees (600) signed majority of the requests. The specialists signed only 210 forms.

\begin{tabular}{|l|c|}
\hline \multicolumn{1}{|c|}{ Fields } & Filled \\
\hline Age & 1488 \\
\hline Unit/Address & 1476 \\
\hline OPD/WARD & 931 \\
\hline Clinical Notes & 1009 \\
\hline Date & 213 \\
\hline Lab From used instead of RRF & 39 \\
\hline
\end{tabular}

Table-ll. Results of filled performas $(n=1500)$

\begin{tabular}{|l|c|}
\hline \multicolumn{1}{|c|}{ Signing Dr } & Number \\
\hline Specialists & 210 \\
\hline MOs & 690 \\
\hline PGs/HOs & 600 \\
\hline Name stamp & 710 \\
\hline MO stamp & 213 \\
\hline No Stamp & 59 \\
\hline Stamp with Tel \# & 01 \\
\hline \multicolumn{2}{|c|}{ Table-III. Results of requesting doctors } \\
\hline
\end{tabular}

\section{DISCUSSION}

Any approved doctor can initiate a radiology request. The completed request form is delivered to the radiology department. On receipt of the request an appropriate time for the examination is decided depending on the degree of urgency indicated on the request i.e. urgent, semi-urgent, and routine.

A radiology request is a clinical document completed by a physician stating what procedure or examination is desired. This document tells why the examination is to be conducted, and on whom the examination will be performed. Also, inherent in the radiology request form is the clinical questions that needs to be answered. A properly completed request form is essential to enhance the efficiency of the radiology 
requesting process. Pertinent identifiers such as name and age are necessary in a proper request form. Adequate clinical history is needed for the radiologist to make the proper clinical diagnosis. Royal College of Radiologists (RCR) Guidelines demands that request forms should be completed accurately and legibly in order to avoid any misinterpretation. Reasons for the request should be clearly stated and sufficient clinical details should be supplied to enable the imaging specialist to understand the particular diagnostic or clinical problems ${ }^{4}$.

All request forms have clearly marked fields, for ease of completion by the requesting physician as well as ample space for pertinent clinical history. The request form must be completely filled out for the examination to be conducted. The main components are:

\section{Patient Data}

$\begin{array}{ll}\text { - } & \text { Full patient name (spelled correctly) } \\ \text { - } & \text { Personal/ CNIC number } \\ \text { - } & \text { Age } \\ \text { - } & \text { Sex } \\ \text { - } & \text { Nocation e.g. ward, private, OPD etc } \\ \text { - } & \text { Date of request } \\ \text { Clinical details } \\ \text { - } \quad \text { Relevant clinical history } \\ \text { - } & \text { Examination requested }\end{array}$

In addition to pertinent patient identifiers and the examination to be conducted, the radiology request must contain clinical information to justify conducting a radiologic examination. This allows the radiologist to make a more accurate and specific diagnosis. If a diagnosis is not known, clinical symptoms such as cough, nausea or headache, etc. are mentioned. Perhaps the most important content in the radiology request is the clinical question to be answered. This question may be direct or indirect. It is imperative that the radiologist identifies this inherent question and seeks to provide the answer through the examination findings.

Examples of clinical data that imply such questions include:

1. The patient's clinical signs and symptoms and requesting diagnosis from the radiologist.

2. An attending physician providing an initial diagnosis and the radiologist confirming the initial diagnosis or otherwise.

3. A surgeon requesting the exact size and location of a mass for surgical planning.

Design of Radiology Request Form (RRF) is more important than its size. A redesigned referral form having specific questions is a better option to collect more information. A properly designed form provides more useful clinical information in less space. The better clinical information helps the radiologist to produce an improved report ${ }^{5}$.

The paper request form serves as the primary means of communication between referring physicians and the radiology department. The clinical information provided on the requisition form, is too often illegible, inadequate, or absent. The computer based request entry system can offer several advantages over the conventional paper requisition form. The physician can enter the necessary information rapidly and easily. The request is transmitted directly to the radiology department and one cannot lose it en route. In a teaching hospital, where several teams of physicians may care for one patient, a centralized entry system can prevent duplicate requests ${ }^{6}$.

To properly determine a protocol for imaging studies and interpret them, radiologists require accurate clinical and laboratory information. Yet the clinical history provided with requisitions is often insufficient, inaccurate, or misleading. A health Summary is a computer-based solution to this problem. Health summary derived from the hospital's database contains imaging reports, laboratory values, 
discharge summaries, patient medication lists and allergy histories?.

It is very unusual to see an ideally filled form in our practice.

An incompletely filled radiology request form (RRF) is a common problem faced by both radiologists and radiographers ${ }^{8}$.

Everyday we deal with forms that have hardly any clinical details and sometimes the handwriting is illegible. Incomplete request forms may result in the following:

- A patient may receive radiation unnecessarily.

- A patient may receive an inappropriate test due to missing clinical information.

- $\quad$ Report is misdirected to wrong ward.

Pertinent clinical information allows the radiologist to tailor the study to the patients' status. If important clinical information is not provided an unwarranted radiology study may be conducted. This can slow down the assessment and treatment of the patient and incurs unnecessary cost to the patient and medical institution. Imaging cost is the amount of money that it costs a hospital to perform a diagnostic procedure. Equipment cost and personnel cost make up the total cost of a diagnostic procedure. By following proper procedure in ordering a radiology examination, the medical institution can save a lot of money each year.

\section{CONCLUSIONS AND RECOMMENDATIONS}

The inadequate transmission of clinical information and the large number of not fully filled request forms observed in this study are typical examples of the various problems that radiologists have to face ${ }^{9}$.

Optimal patient care demands that remedial action be taken in order to change the current inadequate radiology referral process. Discussions on the possible actions to reach this goal, led to the following list of suggestions. ${ }^{10}$.

- $\quad$ An inter office note (ION) to the medical superintendent, consultants, senior registrars and house officers elucidating the above findings and stressing the need to change current practice.

- Instructions to radiological staff to return any inadequately completed forms at a stage BEFORE these are actually recorded in the department's database.

- $\quad$ Returning of request forms is to be done with great care in order to avoid any unwanted delays of urgent examinations and above all any patient suffering, whilst ensuring safe practice.

- Delivering lectures to the young doctors on the importance of properly filling radiology request forms ${ }^{11}$.

- $\quad$ At times, doctor just signs the radiology request form and the nursing staff do filling of form. The signing physician himself should personally fill all the fields.

- $\quad$ Name of patient should be written in Urdu as well as English to avoid confusion.

- $\quad$ Applying necessary changes to the current request forms, ensuring that adequate spacing is provided for the required fields.

- $\quad$ Radiology departments should be involved in the orientation programs for new house officers and resident doctors to let them know the importance of various items on the request forms ${ }^{12}$.

- With the increasing concerns on radiation exposure and its effects, it is more and more important that form is filled with due responsibility. All this is possible if the small piece of paper is treated with respect.

Copyright@ 17 Dec, 2012. 


\section{REFERENCES}

1. Mervyn D. Cohen, Khurshaid Alam. Radiology clinical synopsis: a simple solution for obtaining an adequate clinical history for the accurate reporting of imaging studies on patients in intensive care units -Pediatr Radiol (2005) 35; 918-922.

2. M. Cohen, S. Curtin, R. Lee. Evaluation of the Quality of Radiology Requisitions for Intensive Care Unit Patients-Academic Radiology, February 2006; 13(2): 236-240.

3. Abdus Salam, Ghulam Sabir Iqbal, Muhammad Usman Ahmed. An analysis of one thousand chest radiographs at a secondary care center -Rawal Med J Jul - Dec 2008; 33(2):150-3.

4. UK Royal College of Radiologist Guidelines, 4th Edition (1998).

5. The Royal College of Radiologists-Making the best use of a department of clinical radiology 2007.

6. MA Wilson. Improvement in referral practices elicited by a redesigned request format-- Radiology, 1983; 146: 677-679.

7. Charles E. Kahn, Jr. Pete G. Kovatsis, Richard N. Messer smith, and James L. Lehr. Automated Entry of Radiology Requisition Information with ArtificialIntelligence Techniques-AJR November 1989; 153:1085-1088.
8. David M. Schuster1 and M. Elon Gale. Computers in Radiology The Malady of Incomplete, Inadequate, and Inaccurate Radiology Requisition Histories: A Computerized Treatment AJR, October 1996; 167: 855859.

9. D. Oswal, D. Sapherson, and A. Rehman. A study of adequacy of completion of radiology request forms. Radiography August 2009; 15(3): 209-213.

10. Ch. Triantopoulou, I. Tsalafoutas, P. Maniatis, D. Papavdis, G. Raios, I. Siafas, S. Velonakis, E. Koulentianos. Analysis of radiological examination request forms in conjunction with justification of $X$ ray exposures European Journal of Radiology, February 2005; 53(2):306-311.

11. Ruben Depasquale, Malcolm P Crockford Ruben Depasquale MD--Are Radiology Request Forms Adequately Filled In?--An Audit Assessing Local Practice-- Malta Medical Journal November 2005; 17 (4): 18-21.

12. Allan Andi, Dr David Howlet; The radiology request form: a guide for the foundation year doctor. British Journal of Hospital Medicine, 08 Oct 2008: 160 -162.

13. SB Adebayo, G0G Awosanya, BO Balogun, A Osibogun. Multicentre Assessment of Radiology Request in South-West Nigeria-- Nigerian Hospital Practice ,2009;3(6).
AUTHOR(S):

1. DR. ABDUS SALAM, MBBS, MCPS, FCPS

Consultant Radiologist,

Rawal Institute of Health Sciences, Khanna Dak, Islamabad.

2. DR. SAIF-UD-DIN SAIF, MBBS, MPH

Assistant Professor/Head of Community Medicine Department Rawal Institute of Health Sciences, Khanna Dak, Islamabad.
Correspondence address:

Dr. Saif-Ud-Din Saif, MBBS, MPH

Assistant Professor/Head of Community Medicine Department Rawal Institute of Health Sciences, Khanna Dak, Islamabad. saifdin75@yahoo.com 